\title{
Photoacids in biochemical applications
}

\author{
Heike Kagel, Marcus Frohme* and Jörn Glökler \\ Molecular Biotechnology and Functional Genomics, Technical University of Applied Sciences, \\ Wildau, Germany
}

\begin{abstract}
.
BACKGROUND: After excitation with light photoacids can change the $\mathrm{pH}$ in a solution by release of a proton. They have been used mostly for excited state proton transfer studies. In this review the general functionality and mechanisms and the subdivision of photoacids is explained.

STATE OF THE ART: Different uses of photoacids are described, covering a wide range of various biochemical topics, focusing on biochemical applications. Examples for the introduced subdivisions are covered.

CONCLUSIONS AND OUTLOOK: The areas in which photoacids can be employed are diverse. Photoacids have a promising future in biotechnology and biochemistry and should be considered for upcoming applications, especially in non-invasive control of biochemical reactions.
\end{abstract}

Keywords: Photoacids, metastable photoacids, light induced, $\mathrm{pH}$ jump, HPTS

$\begin{array}{ll}\text { Abbreviations } \\ \text { ESPT } & \text { excited state proton transfer } \\ \text { PAG } & \text { photoacid generator } \\ \text { PAH } & \text { photoacid } \\ \text { mPAH } & \text { metastable photoacid } \\ \text { HPTS } & \text { 8-Hydroxypyrene-1,3,6-Trisulfonic Acid } \\ \text { MIC } & \text { minimum inhibitory concentration } \\ \text { CF-MUP } & \text { 6-chloro-8-fluoro-4-methylumbelliferone phosphate } \\ \text { MGCB } & \text { Malachite Green Carbinol Base }\end{array}$

\section{Background}

\subsection{Photoacidity}

A photoacid is an aromatic alcohol that transforms into a strong acid upon irradiation [1] and undergoes excited state proton transfer (ESPT) in this process [2-4]. The acidity enhancement after excitation is typically on a scale of a factor of $10^{6}-10^{8}$, thus decreasing the $\boldsymbol{p} \boldsymbol{K}_{\boldsymbol{a}}$ by $6-8$ units [5]. Photoacidity was first investigated by Förster and Weller, more than half a century ago [6] and is best described by the Förster cycle diagram (Fig. 1). Here, $\mathrm{ROH}$ is the protonated form of the photoacid in the ground state, excited to the protonated photoacid $\mathbf{R} * \mathbf{O H}$ which dissociates to form an ion pair

\footnotetext{
${ }^{*}$ Corresponding author: Prof. Dr. Marcus Frohme, TUAS Wildau, Hochschulring 1, 15745 Wildau, Germany. Tel.: +49 3375508 249; E-mail: marcus.frohme@th-wildau.de.
} 


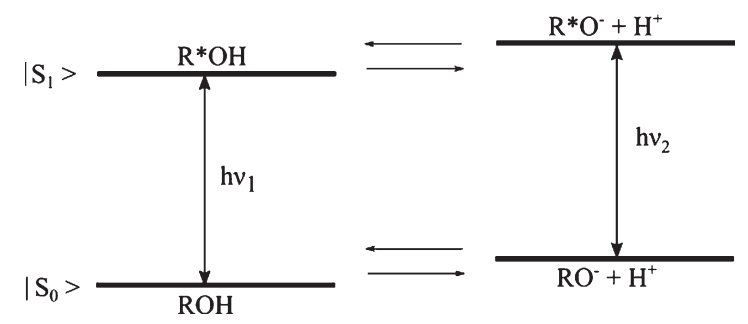

Fig. 1. Schematic representation of a photoacid's energy level, where $\mathrm{R} * \mathrm{OH}$ is the excited photoacid with its conjugate base $\mathrm{R} * \mathrm{O}-, \mid \mathrm{S}_{1}$ represents the first singlet excited state and $\mid \mathrm{S}_{0}$ the ground state.

with the proton. The proton can perform geminate recombination with the excited deprotonated form of the photoacid $\left(\mathbf{R}^{*} \mathbf{O}^{-}\right)$[7] or diffuse away from the parent ion [8].

According to Förster, photoacidity is defined in terms of $K_{a}^{*}$, the excited state equilibrium constant for the dissociation reaction of the photoacid [9]:

$$
\Delta p K_{a}=p K_{a}-p K_{a}^{*}
$$

$\boldsymbol{p} \boldsymbol{K}_{\boldsymbol{a}}^{*}$ being the equilibrium constant in the excited State $S_{1}$ and $\boldsymbol{p} \boldsymbol{K}_{\boldsymbol{a}}$ being the equilibrium constant for the proton dissociation reaction in the ground state $S_{0}$, as it is valid for proton transfer reactions in water: $\boldsymbol{H A}+\boldsymbol{H}_{2} \boldsymbol{O} \leftrightarrow \boldsymbol{H}_{3} \boldsymbol{O}^{+}+\boldsymbol{A}^{-}$, HA being the Brønsted Acid, which donates a proton $H^{+}$to the solvent such as water. In this process an anion $A^{-}$is produced [10]. Thus, a photoacid can also be defined as a molecule, in which the ground state acidity is lower than its acidity in the exited state $p K_{a}^{*}<p K_{a}$. Therefore, the higher the $\Delta p K_{a}$, the stronger the photoacid. So far, the majority of research focused on dynamical studies of acid-base reactions by using these unique properties of photoacids $[3,7,11]$. Generally, photoacids are subdivided into two main classes: Photoacid generators (PAGs) and reversible photoacids (PAHs).

\subsection{Photoacid generators and photoacids}

While PAGs irreversibly undergo proton photo dissociation, PAHs reversibly recombine after the photo induced dissociation, when the irradiation is terminated.

PAGs dissociate upon irradiation and irreversibly form strong acids while undergoing a photo destructive process. This unique property to acidify the surrounding upon irradiation has often been used in photo polymerization; for example to enhance polymer properties, [12, 13], to start polymerization reactions [14] or in lithography [15].

In contrast to a PAG, a reversible photoacid is not structurally disintegrated upon illumination, but thermally re-associates with a proton after returning to the ground state. Very common families of photoacids include phenol, naphthol and pyrene derivates, as illustrated in Fig. 2. Characteristic is the functional group for all reversible photo acids, which donates the proton in the ESPT process [10] and is able to interact with the surrounding. Depending on various factors the photo induced freed proton can diffuse and escape the zone of influence of the parent ion, enabling it to react with the surrounding environment. It is also possible that it is recaptured by the parent ion after this has returned back to the ground state $S_{0}$. The photo induced dissociation can be induced by a laser pulse, converting a weak acid into a strong acid [16-18]. This process takes place on the scale of pico-to nanoseconds. 
<smiles>Oc1ccccc1</smiles>

Phenols<smiles>Oc1cccc2ccccc12</smiles>

Naphthols<smiles>Oc1ccc2ccc3cccc4ccc1c2c34</smiles>

Pyrenes

Fig. 2. Families of photoacids - crucial for the mechanism to work is the functional $\mathrm{OH}$ group, which donates the $\mathrm{H}^{+}$upon irradiation.
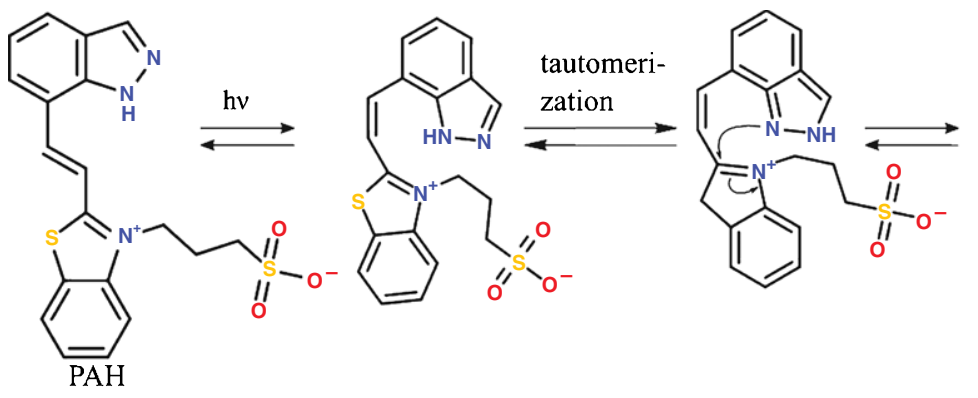
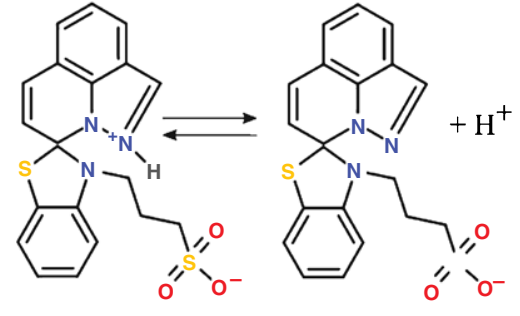

$\mathrm{PA}^{-}$

Fig. 3. Proposed multi- stepped mechanism by Abeyrathna et al. [1] of the metastable photoacid mPAH 2.

\subsection{Metastable photoacids}

Metastable photoacids (mPAH) present a new opportunity regarding photo induced $\mathrm{pH}$ jumps. A rising interest in the development and design of new photoacids suitable for biotechnology and biochemistry is also demonstrated by Liao, who published a paper on how to design mPAHs [1]. While the irradiation of a conventional PAH results in very short-lived $\mathrm{pH}$ jumps in the order of nano-to microseconds until recombination of the proton with the parent ion, metastable photoacids can change the $\mathrm{pH}$ for seconds up to hours [19]. The long lasting $\mathrm{pH}$ jump is correlated to the design of the mPAHs: They are generally designed by linking an electron-accepting moiety and a weakly acidic nucleophilic moiety with a double bond, which enables absorption of visible light [1]. As both forward and backward reactions are multi-stepped the half live is extended [20]. A schematic sketch of the proposed mechanism is displayed in Fig. 3.

It is worth mentioning that there are also metastable photobases, which principally function similar to metastable photoacids: They donate an $\mathrm{OH}^{-}$and thus increase the $\mathrm{pH}$ value of a solution [21].

\section{State of the art}

\subsection{Photoinduced $\mathrm{pH}$ jumps}

A famous representative of the PAHs is pyranine, i.e. 8-hydroxy-1,3,6-pyrenetrisulfonate or HPTS $[11,22]$. Not only has HPTS been extensively employed in ESPT studies [10], but also in biological 
applications to monitor the $\mathrm{pH}$ in cells by fluorescence in near neutral $\mathrm{pH}$ [23]. It has also been used to develop a sterilizeable HPTS- based sensor, which can be applied for online $\mathrm{pH}$ monitoring of $E$. coli fermentation [24]. Gutman et al. demonstrated [16, 18] that aqueous solutions could be acidified on a microsecond scale by exciting the photo acid HPTS, using a short laser pulse of $50 \mathrm{~ns}$. Also Hynes et al. demonstrated the photochemical proton transfer in aqueous solution using HPTS, phenol and substituted phenols [25]. pH indicators, such as bromcresol green, were used for a secondorder diffusion controlled reaction: The photoinduced proton ejected from the photoacid changed the formation of the $\mathrm{pH}$ indicator and made a spectroscopic determination of $\mathrm{pH}$ change possible. Also using HPTS, Haghighat et al. could measure an increase in proton conductivity upon irradiation of photo acid doped materials with the photoacid [26]. Thus demonstrating, that the proton released is indeed liberated and can interact with its surrounding.

A longer $\mathrm{pH}$ change $(\sim 0.1 \mathrm{~s})$ was demonstrated using a synthesized 2-naphthol derivate by Nunes et al. [27]. However, the used photoacid is excited at $266 \mathrm{~nm}$, making it unsuitable for biological applications as most biological components such as nucleic acids are damaged by applying this UV light. Also many analytical methods in biology are based on fluorescence assays, which will be obscured by a photoacid's strong fluorescence.

Yang et al. demonstrated the color change in polymer films doped with $\mathrm{pH}$ indicators such as bromcresol green, using an mPAH [28]. The photoswitching of a $\mathrm{pH}$ indicator using another mPAH, has also been achieved by Vallet et al. [29]. Another $\mathrm{pH}$ switching experiment has been conducted by Shi et al., shrinking a pH sensitive hydrogel, by irradiating it in a solution containing an mPAH [30]. In a similar experiment Chen et al. demonstrated the color change of $\mathrm{pH}$ indicators in solution together with an $\mathrm{mPAH}$ [31]. Not only reversible $\mathrm{pH}$ decreases, but also a light induced $\mathrm{pH}$ increase of aqueous solutions could be achieved by Masahiro using a photobase [21].

\subsection{Applications in biochemistry}

A wide range of enzymes have different activities, strongly depending on the $\mathrm{pH}$, thus creating a condition by which enzyme activity can be optically controlled by a light pulse. Kohse et al. succeeded in optical control of acid phosphatase activity by a laser-induced $\mathrm{pH}$ drop [32]. This allowed the development of a non-invasive initiation of a biochemical reaction. Acid phosphatase was combined with a PAG ( 2 nitrobenzaldehyde) in an aqueous solution. Since the enzyme is active at a pH below 8 and has its optimum activity at a $\mathrm{pH}$ of 5 , the hydrolytic activity of acid phosphatase was controlled by switching the conditions from an alkaline $\mathrm{pH}$ of 8 to an acidic $\mathrm{pH}$ of 6 . To yield a $\mathrm{pH}$-jump of nearly two units, a short laser pulse was used, inducing the proton release of the PAG. Typical sample irradiation times were $250 \mathrm{~ms}$, lowering the $\mathrm{pH}$ sufficiently to activate the enzyme. In order to monitor the altered activity 6-chloro-8-fluoro-4-methylumbelliferone phosphate (CF-MUP) was used as a substrate. CF-MUP significantly changes its absorption at $360 \mathrm{~nm}$ after hydrolysis from CF-MUP to 6-chloro-8-fluoro-4-methylumbelliferone (CF-MU) [32, 33]. CF-MUP was also used by Yang et al. to track the activity of acid phosphatase [33]. Kohse et al. arrived at an experimental setup without any loss of enzyme activity. Thus, it is possible to locally change the $\mathrm{pH}$ rapidly in a very small volume, even controlling enzymatic activities in an optical, non-invasive manner without loss of activity. Other promising enzymes with a significant $\mathrm{pH}$-profile concerning activity are suggested. Those could possibly be activated in a similar manner, offering a wide range of applications. Examples are: Laccase, catalase, polyphenol oxidase, $\beta$-glucosidase, L-arginine decarboxylase [32]. Since PAGs are used, these reactions are all irreversible.

A prototypic example for a bioelectrical, miniaturizable device using a photoacid is given by PeretzSoroka et al. [34]. They developed a prototype with which they could control and monitor the $\mathrm{pH}$ using a nanowire-based FET device. Silicon nanowires were chemically modified by photoactive HPTS 
derivatives and by biomolecules, e.g. enzymes. All of the involved compounds were immobilized to the surface. Effected by the surface-anchored photoacid molecules, a photo induced $\mathrm{pH}$ jump could be detected resulting in a proton transfer to the solvent. This was achieved by measuring the change in surface potential alteration which modulates the current flow. HPTS exhibits a pH-dependent adsorption shift and therefore additionally allowed the $\mathrm{pH}$ determination by ratiometric fluorescence measurements in parallel: Two excitation wavelengths at $405 \mathrm{~nm}$ and $450 \mathrm{~nm}$ have been used. At a pH below 7 the absorbance at $450 \mathrm{~nm}$ rapidly decreases and thus the fluorescence [35]. Using this characteristic of HPTS, both as a photoacid and as a fluorescence $\mathrm{pH}$ indicator Peretz-Soroka et al. performed the experiment using HPTS and trypsin modified silicon nanowires. Trypsin acidifies the solution while hydrolyzing its specific substrate $\mathrm{N} \alpha$-Benzoyl-L-arginine ethyl ester (BAEE), thus resulting in a longterm change in conductivity and $\mathrm{pH}$. The enzyme activity was monitored using the described device and method above [34]. As a next step a HPTS-pepsinogen complex was anchored to the silicon nanowires. The inactive proenzyme pepsinogen is activated at an acidic $\mathrm{pH}$ of about $2-4$ by selfprocessing resulting in the active protease pepsin. In addition, the reaction leads to further acidification of its surrounding by the hydrolysis reaction [36]. By steady HPTS illumination using a wavelength of $400 \mathrm{~nm}$ a $\mathrm{pH}$ jump was caused. The generation of the active pepsin was controlled and monitored with a single multifunctional device. Therefore, local modulation and monitoring of surface $\mathrm{pH}$ by the use of a reversible photoacid was successfully achieved, offering the development of multifunctional bio- FETs [37].

Photoacids cannot only induce $\mathrm{pH}$ jumps, but are also suitable to analyze biomaterials, their proton transfer rates, as well as their binding characteristics. Amdursky et al. used surface-bound photoacids to study excited-state proton transfer and binding sites on insulin amyloid fibril surfaces [8, 10]. Diseases, such as Alzheimer or Parkinson are related to the formation of amyloid fibrils [38, 39]. During the fibrillogenesis process, the soluble amyloid proteins aggregate into an insoluble structure. It is important to fully understand the structure of amyloid fibrils to be able to design molecular inhibitors to fibrillogonesis [40,41]. This can be supported by analysis of the fibrillar surface structure and their interaction with aqueous surrounding. The use of photoacids of different strength allowed to analyze the propagation of protons along the fibril surface in order to explore various binding sites of insulin amyloid fibril. Also, using this strategy photoacids can be used as a marker for amyloid fibrils, employing their fluorescence upon excitation. The group used photo acids of different strength: The naphthol- based photoacids strongly differed in their $p K_{a}$, thus releasing a significantly different proton concentration upon irradiation. Also the selected naphthol derivates differed in their sulfonic groups and thus in their binding capacity. Followed by extensive molecular dynamics simulations, the binding modes and mechanisms of the surface-bound photoacids were analyzed, resulting in a detailed characterization of different binding modes to the surface of amyloid fibrils. The results Amdursky et al. could contribute to the design of fibrillogenesis inhibitors. Hence, photoacids can also be applied as fluorescent markers to understand the binding mechanism of small molecules, such as photoacids, to amyloid fibrils.

A strategy for decreasing adverse side effects of the antibiotic colistin in clinical environments was proposed by Luo et al. [42]. They conducted an experiment, irradiating multi-drug resistant bacteria, with visible light. Pseudomonas aeruginosa is a main cause of hospital infections and is difficult to treat because of the wide spread multidrug resistant strains [42], although colistin serves as an effective measure against multidrug resistant bacteria [43]. However, it exhibits serious nephrotoxicity and neurotoxicity [44]. In the attempt to decrease the amount needed to effectively inactivate bacteria, colistin was used in combination with mPAH [42]. When illuminated with visible light, $470 \mathrm{~nm} \mathrm{mPAH}$ releases protons and thus lowering the $\mathrm{pH}$, recombining again with the donated proton when the light is switched off. Simple commercially available LEDs were used to excite the photoacid and the $\mathrm{pH}$ could be decreased from 5.8 to 3.3. When the light was turned off the $\mathrm{pH}$ first rapidly $(70 \mathrm{~s})$ increased 
to 4.7 and gradually ( $\sim 5 \mathrm{~min}$ ) back to to 5.8 . Although the mPAH has a very low toxicity to cells, it was demonstrated that they had a slight antibacterial activity upon illumination, due to the reduction of $\mathrm{pH}$. However, it was clearly shown that only the illumination of the $\mathrm{mPAH} /$ colistin combination had the effect of significantly lowering the minimum inhibitory concentration (MIC) of colistin needed in order to kill the multidrug resistant Pseudomonas aeruginosa by 32 times. These results open a promising approach in killing multidrug resistant bacteria in which photoacids could be used in the design of new drug formulations or drug carriers.

Photoacids are still mostly used for theoretical modeling, e.g. studying the ESPT in physics and chemistry. Those experiments were often conducted in biologically unfavorable media (e.g. DMSO, methanol) or conditions (very low $\mathrm{pH}$ values). Driven by the desire to open up opportunities for mPAHs in the biological field, yet another metastable photoacid was developed, which is able to keep its proton in PBS buffer with a physiological pH of 7.4 [20]. The UV- Vis spectrum of this mPAH was studied in PBS buffer at a $\mathrm{pH}$ of 7.4. A strong absorption band at $422 \mathrm{~nm}$ was observed in the protonated form of the mPAH, decreasing when adding $\mathrm{NaOH}$ and thus deprotonating the mPAH. The photoreaction was also studied by NMR analysis after 10 minutes irradiation. The changes in the NMR spectrum confirmed, that a deprotonation process indeed occurs upon irradiation. Furthermore, the deprotonation of the excited mPAH was also demonstrated using an acridine dye, which changed from orange do blue upon light-induced protonation. All the studies performed on the mPAH clearly prove that the multistepped conformation change indeed takes place, resulting in the release of a proton upon illumination. In addition, a reversible reaction was demonstrated as well [20]. Yet, another advantage of this newly developed mPAH is that it is excitable with visible light - e.g. commercially available LEDs and not UV- light which will likely damage biological compounds. After irradiation, the mPAH reached its original $\mathrm{pH}$ only after being kept in the dark for $48 \mathrm{~h}$, which is still too long time for most biological applications. Nevertheless, it exemplifies the motivation to develop new photoacids that are suitable for biological applications.

\section{Conclusions and outlook}

As highlighted in this review, there have been several reports on the application of photoacids in biochemistry. Photoacids offer a local control of $\mathrm{pH}$, making them extremely attractive for miniaturizable, non-invasive and time-resolved control of reactions applications in biochemistry. The possible applications of photoacids in the biological field are large: While some of the efforts summarized in this review focus on controlling enzyme activity, others have developed methods do design bioelectrical devices to control and monitor the $\mathrm{pH}$, or analyze structures. Applications based on photoacids, for example to remotely control the $\mathrm{pH}$ value in solution to switch an enzyme, are gaining in significance. Hence, photoacids in biochemistry or biology can be considered a novel area of research and display a great potential for future development. Apart from optically controlling enzyme activity, photoacids were also used to develop new multifunctional bioFETs for controlling and monitoring $\mathrm{pH}$ value simultaneously. The small molecules were also used to study biomaterial characteristics, aiding in creating inhibitors for e.g. Alzheimer's disease. The versatility of photoacids in biochemical applications becomes apparent by the examples compiled in this review.

Photoacids have the potential for high throughput methods and automation. It is also possible to excite photoacids using commonly available LEDs, making their use in devices and instruments more attractive. Especially, novel concepts for biosensors and screening platforms based on photoacids are beginning to emerge. 


\section{Conflict of interest}

The authors have no financial, consulting or personal relationships with other persons or organizations which could have influenced the present work.

\section{Acknowledgments}

The authors acknowledge the financial support by the Europäischer Fonds für regionale Entwicklung (EFRE) and the Investitionsbank des Landes Brandenburg (ILB). The work of the authors Heike Kagel and Jörn Glökler is financed within the program StaF from the Ministry of Science, Research and Culture (MWFK) of the federal state of Brandenburg (Germany) under the RefNo. 85000780.

\section{References}

[1] Liao Y. Design and Applications of Metastable-State Photoacids. Accounts of Chemical Research. 2017;50(8):1956-64. PMID: 28692282.

[2] Tolbert LM, Solntsev KM. Excited-State Proton Transfer: From Constrained Systems to Super Photoacids to Superfast Proton Transfer. Accounts of Chemical Research. 2002;35(1):19-27. PMID: 11790085.

[3] Monica Barroso LGA, Formosinho SJ. Intersecting-state model claulations on fast and ultrafst excited-state proton transfers in naphthols and subsitiuted naphthols. Journal of Photochemistry and Photobiology. 2002;154.

[4] Arnaut LG, Formosinho SJ. Excited-State proton Transfer Reations I. Fundamentals and intermolecular reactions. 1993.

[5] Rini M. Femtosecond Mid-Infrared Spectroscopy of Elementary Photoinduced Reactions; 2003.

[6] A W. Fast reactions of excited molecules. Prog React Kinet Mech. 1961.

[7] Noam Agmon EP, Huppert D. Geminate recombination in proton transfer reactions. II. Comparison of diffusional and kinetic schemes. The Journal of Chemical Physics. 1988;88(9):5631-8.

[8] Amdursky N, Rashid MH, Stevens MM, Yarovsky I. Exploring the binding sites and proton diffusion on insulin amyloid fibril surfaces by naphthol-based photoacid fluorescence and molecular simulations. Scientific Reports. 2017;7(1):6245.

[9] Wiley J, Sons. The chemistry of phenols. John Wiley and Sons; 2003.

[10] Amdursky N, Simkovitch R, Huppert D. Excited-State Proton Transfer of Photoacids Adsorbed on Biomaterials. The Journal of Physical Chemistry B. 2014;118(48):13859-69. PMID: 25380297.

[11] Tran-Thi, Gustavsson, Prayer, Pommeret, Hynes. Primary ultrafast events preceding the photoinduced proton transfer from pyranine to water. Chemical Physics Letters. 2000;329(56):421-30.

[12] Anger C. Photosäure induzierte Polymerisation von Oxasilacyclen zum Aufbau neuer Polysiloxan Architekturen. Technische University Munich; 2013.

[13] Johns VK, Patel PK, Hassett S, Calvo-Marzal P, Qin Y, Chumbimuni-Torres KY. Visible Light Activated Ion Sensing Using a Photoacid Polymer for Calcium Detection. Analytical Chemistry. 2014;86(13):6184-7. PMID: 24893213.

[14] Klikovits N, Knaack P, Bomze D, Krossing I, Liska R. Novel photoacid generators for cationic photopolymerization. Polym Chem. 2017;8:4414-21.

[15] Ayothi R, Yi, Cao HB, Yueh W, Putna S, Ober CK. Arylonium Photoacid Generators Containing Environmentally Compatible Aryloxyperfluoroalkanesulfonate Groups. Chemistry of Materials. 2007;19(6):1434-44.

[16] Gutman M, Huppert D, Pines E. The pH jump: A rapid modulation of $\mathrm{pH}$ of aqueous solutions by a laser pulse. Journal of the American Chemical Society. 1981;103(13):3709-13.

[17] Warshel A. Picosecond Studies of Excited-State protonation and Deprotonation Kinetics. The Laser pH Jump. Journal of the American Chemical Society. 1979.

[18] Gutman M, Nachliel E, Gershon E, Giniger R, Pines E. pH Jump: Kinetic Analysis and Determination of the DifussionControlled Rate Constants. 1983.

[19] Liao Y. Photoacid compositions having extended lifetime of proton dissociation state. Google Patents; 2016. US Patent App. 15/057,856.

[20] Abeyrathna N, Liao Y. A Reversible Photoacid Functioning in PBS Buffer under Visible Light. Journal of the American Chemical Society. 2015.

[21] Masahiro I. Light-induced Reversible pH Change. Journal of American Chemical Society. 1983. 
[22] Spry DB, Goun A, Fayer MD. Deprotonation Dynamics and Stokes Shift of Pyranine (HPTS). The Journal of Physical Chemistry A. 2007;111(2):230-7. PMID: 17214458.

[23] Wolfbeis OS, Faerlinger E, Kroneis H, Marsoner H. Fluorimetric analysis. Fresenius' Zeitschrift für analytische Chemie. 1983;314(2):119-24.

[24] Kermis HR, Kostov Y, Harms P, Rao G. Dual Excitation Ratiometric Fluorescent pH Sensor for Noninvasive Bioprocess Monitoring: Development and Application. 2002.

[25] Hynes J, hoa Tran-thi T, Granucci G. Intermolecular photochemical proton transfer in solution: New insights and perspectives. 2002.

[26] Haghighat S, Ostresh S, Dawlaty JM. Controlling Proton Conductivity with Light: A Scheme Based on Photoacid Doping of Materials. The Journal of Physical Chemistry B. 2016;120(5):1002-7. PMID: 26771862.

[27] Nunes RMD, Pineiro M, Arnaut LG. Photoacid for Extremely Long-Lived and Reversible pH - Jumps. Journal of the American Chemical Society. 2009;131(26):9456-62. PMID: 19518094.

[28] Yang C, Khalil T, Liao Y. Photocontrolled proton transfer in solution and polymers using a novel photoacid with strong C-H acidity. RSC Adv. 2016;6:85420-6.

[29] Vallet J, Micheau JC, Coudret C. Switching a pH indicator by a reversible photoacid: A quantitative analysis of a new two-component photochromic system. 2015.

[30] Shi Z, Peng P, Strohecker D, Liao Y. Long-Lived Photoacid Based upon a Photochromic Reaction. Journal of the American Chemical Society. 2011;133(37):14699-703. PMID: 21823603.

[31] Hongbin Chen YL. Photochromism based on reversible proton transfer. Journal of Photochemistry and Photobiology A: Chemistry. 2015.

[32] Kohse S, Neubauer A, Pazidis A, Lochbrunner S, Kragl U. Photoswitching of Enzyme Activity by Laser-Induced pH-Jump. Journal of the American Chemical Society. 2013 05;135.

[33] Yang D, Li Z, Allan Diwu Y, Fu H, Liao J, Wei C, et al. A Novel Fluorogenic Coumarin Substrate for Monitoring Acid Phosphatase Activity at Low pH Environment; 2008.

[34] Tripathi RKD, DN. A study of thermal denaturation /renaturatoin in DNA using laser light scattering: A new approach. Indian J Biochem Biophys. 2005;42.

[35] Slyusareva EA, Gerasimova MA. pH-Dependence of the Absorption and Fluorescent Properties of Fluorone Dyes in Aqueous Solutions. Russian Physics Journal. 2014;56(12):1370-7.

[36] Piper DW, Fenton BH. pH stability and activity curves of pepsin with special reference to their clinical importance.; 1965.

[37] Peretz-Soroka H, Pevzner A, Davidi G, Naddaka V, Kwiat M, Huppert D, et al. Manipulating and Monitoring On-Surface Biological Reactions by Light-Triggered Local pH Alterations. Nano Letters. 2015;

[38] Chiti F, Dobson CM. Protein Misfolding, Functional Amyloid, and Human Disease. Annual Review of Biochemistry. 2006;75(1):333-66. PMID: 16756495.

[39] Rochet JC, Lansbury PT. Amyloid fibrillogenesis: Themes and variations. Current Opinion in Structural Biology. 2000;10(1):60-8.

[40] Findeis MA, Molineaux SM. Design and testing of inhibitors of fibril formation. In: Amyloid, Prions, and Other Protein Aggregates. vol. 309 of Methods in Enzymology. Academic Press; 1999. pp. 476-88.

[41] Bouchard M, Zurdo J, Nettleton EJ, Dobson CM, Robinson CV. Formation of insulin amyloid fibrils followed by FTIR simultaneously with CD and electron microscopy.; 2000.

[42] Luo Y, Wang C, Peng P, Hossain M, Jiang T, Fu W, et al. Visible light mediated killing of multidrug - resistant bacteria using photoacids. J Mater Chem B. 2013;1:997-1001.

[43] Walkty A, DeCorby M, Nichol K, Karlowsky JA, Hoban DJ, Zhanel GG. In Vitro Activity of Colistin (Polymyxin E) against 3,480 Isolates of Gram-Negative Bacilli Obtained from Patients in Canadian Hospitals in the CANWARD Study, 2007-2008; 2009.

[44] Wallace SJ, Li J, Nation RL, Rayner CR, Taylor D, Middleton D, et al. Subacute toxicity of colistin methanesulfonate in rats: Comparison of various intravenous dosage regimens. Antimicrobial Agents and Chemotherapy. 2008;52: $1159-61$. 